\title{
IGF-1 and IGF-1 SDS - fit for purpose?
}

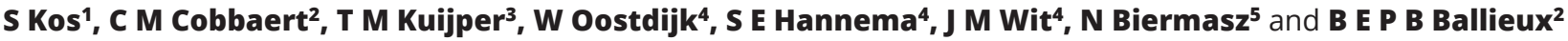

${ }^{1}$ Department of Clinical Chemistry, Maasstad Hospital, Rotterdam, The Netherlands, ${ }^{2}$ Department of Clinical Chemistry and Laboratory Medicine, Leiden University Medical Center, Leiden, The Netherlands, ${ }^{3}$ Department of Science, Maasstad Hospital, Rotterdam, The Netherlands, ${ }^{4}$ Department of Paediatrics, Leiden University Medical Center, Leiden, The Netherlands, and ${ }^{5}$ Division of Endocrinology and Center for Endocrine Tumours, Department of Medicine, Leiden University Medical Center, Leiden, The Netherlands

\section{Dear Editor,}

Three recent publications $(1,2,3)$ have reported that the differences in IGF-1 normative data significantly impact the classification of patients with growth hormone $(\mathrm{GH})$ excess and deficiency disorders in such a way that clinical performance is severely flawed. This concerns not only pediatric but also adult patients. With this letter, we aim to address additional issues, not previously addressed, which add to the complexity of the interpretation of IGF-1 results. Specifically, the relation between analytical performance of IGF-1 assays and acceptable clinical performance is discussed.

Given the strong dependency of serum IGF-1 on age and gender, calculation of the IGF-1 standard deviation score (s.D.-score or SDS) is preferred as an indirect measure of the GH secretory status. However, in a consensus statement on standardization of IGF-1 assays (4), no recommendation on preferred SDS calculation is given. SDS is widely used in children for anthropometric measures, such as length, height, weight, body mass index and head circumference. However, these measures can be measured accurate and precise without variation in time, in contrast to IGF-1 measurements, which show considerable methodological and biological variability.

Two extensive multicenter publications reporting IGF-1 reference values including SDS calculation, stressed the tremendous efforts needed to establish age- and sex-dependent normative data for IGF-1, the limited comparability of IGF-1 assays and the differences between various normative datasets available $(5,6)$. However, despite all efforts, recent reports have shown that variation in IGF-1 normative data still impacts the classification of patients with GH-related disorders, not only in pediatric patients (GH deficiency) but also in adult acromegaly patients $(1,2,3)$.

In a retrospective study, we have applied two SDS mathematical calculations based on two independent normative data sets to 2828 IGF-1 results analyzed using the IDS-iSYS method. These 2828 IGF-1 results were extracted from the laboratory information system and requested during 2015. No clinical data of these patients were collected. The two SDS calculations include those based on the normative data of Bidlingmaier (SDS1) (6) and on a Dutch cohort (SDS2, unpublished observations). The 'Dutch' harmonized reference values are based on samples measured at university hospitals in Utrecht and Amsterdam, using DiaSorin Liaison ${ }^{\circledR}$ XL and Siemens Immulite ${ }^{\circledR} 1000$ harmonized data, consisting of 1500 subjects (695 males and 805 females, 2-75 years of age). There were 207 pediatric samples from 0-18 years (106 males, 101 females), previously addressed in the publication of Broeren et al. (3), used for the LMS curve fitting. In each of the age groups approximately 25 subjects were included. This reference set is far smaller compared to the ten times larger population used for calculation of SDS values according to Bidlingmaier et al. The extremes of the age were extrapolated. The Dutch harmonization program for IGF-I has been implemented in 2013 in analogy to the harmonization of GH measurements in the Netherlands, to reduce between-laboratory variability of IGF-1 at the clinical decision point of $20 \mathrm{mIU} / \mathrm{L}(7,8)$.

The differences were not obvious in the group as a whole, but they were significant when an analysis in groups stratified by age and gender was performed. For example, at age $<10$ years, $10-20$ years and $\geq 40$ years, $13 \%$ compared to $2.3 \%, 7.8 \%$ compared to $4.5 \%$ and https://eje.bioscientifica.com https://doi.org/10.1530/EJE-19-0458 (c) 2019 European Society of Endocrinology Printed in Great Britain
Published by Bioscientifica Ltd. 
$6.4 \%$ compared to $12.9 \%$ males were classified as <-2 S.D., using SDS1 and SDS2 respectively (Fig. 1A). In females, the differences were largest at the age of $\geq 40$ years, as $2.5 \%$ compared to $8.7 \%$ females were classified as $<-2$ s.D., using SDS1 and SDS2, respectively (Fig. 1B). The differences were observed also at +2 s.D. (data not shown). Therefore, we could confirm the findings of aforementioned studies.

In addition to the choice of normative data, other issues, not addressed in the previously mentioned studies, add to the complexity of the interpretation of IGF-1 results. These include (1) defining clinically acceptable variations in IGF-1 results and SDS (commonly referred to

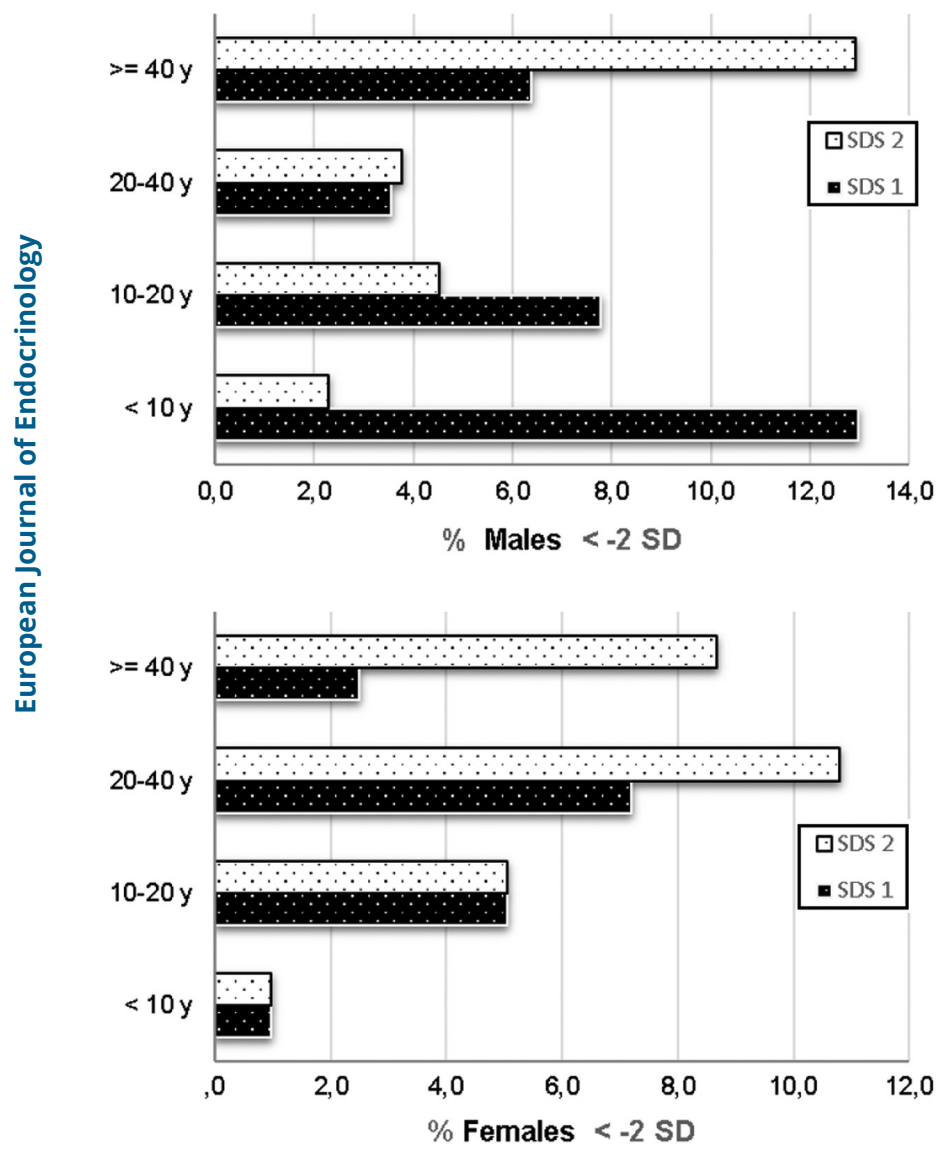

\section{Figure 1}

Comparison of the two normative data sets in the groups stratified by age. Two mathematical calculations based on two independent normative data sets were applied to 2828 IGF-1 results analyzed using the IDS-iSYS method to calculate IGF-1 SDS values. SDS 1: calculation based on the normative data of Bidlingmaier (5); SDS2: calculation based on a Dutch cohort (unpublished). The percentage of individuals from different age groups with an IGF-1 SDS $<-2$ SDS is shown for each normative data set. (A) \% males $<-2$ s.D.; (B) \% females <-2 s.D. as clinical performance specifications (CPS)); (2) defining analytically acceptable limits of variation (usually referred to as analytical performance specifications (APS)) and (3) awareness of the non-linearity of the relationship between the IGF-1 concentration and the SDS.

CPS and APS are interrelated terms, often used in clinical chemistry in different stages of test evaluation under different terminology, but this terminology is recently introduced by the European Federation of Clinical Chemistry (EFLM) Working group 'Test Evaluation' (9). The premise is that APS is guided by CPS, as only clinical context determines the need for more or less strict APS. As CPS for IGF-1 has not been defined yet, we aimed to establish CPS in dialog with experienced (pediatric) endocrinologists. These CPS were formulated in consultation with experienced pediatric endocrinologists (coauthors WO, SH, JMW) and an experienced internist-endocrinologist $(\mathrm{NB})$, representing a team of endocrinologists from Leiden University Medical Center. The proposed CPS were discussed with regional pediatric endocrinologists at a regional meeting and presented at the annual joint Dutch-Belgian Endocrinology Congress ('Dutch Endocrine Meeting, Noordwijkerhout, February 2018'). They were considered 'realistic'. According to some endocrinologists even smaller CPS for s.D. would be preferable (i.e. 0.25 SDS). In this process it was agreed that while a CPS of 0.25 SDS would be desirable, a CPS of 0.5 SDS was still considered clinically acceptable. This implies that an IGF-1 SDS result must have a 95\% confidence interval not exceeding +0.5 and -0.5 SDS. There is however a possibility that experts used to deal with different assays of which some have shown serious drifts over time (10) may have had to adjust their own acceptable CPS dependent on the analytical performance of the assay used, which might have been larger.

The next step was to test the current performance of the IGF-1 assay to this CPS by calculating measurement uncertainty (abbreviation: MU) of the assay according to the ISO 15189 recommendation for medical laboratories. According to the ISO 15189 guideline, medical laboratories are obliged to report MU of their results. MU may be calculated by a straightforward procedure combining long-term imprecision from internal quality control (internal QC) results and bias from external quality controls (EQAs). MU gives an estimation of the long-term uncertainty of a particular test result.

We therefore wished to investigate whether the MU of IGF-1 assay translates into an uncertainty of the IGF-1 SDS of maximally +/-0.5 SDS? MU during the period that the results were obtained was estimated at 22 and 
$16 \%$ in 2017 and 2018, respectively for a large measurement range $(8.7-59.2 \mathrm{nmol} / \mathrm{L})$ based on internal and external quality assessment data. However, at SDS of -2 or +2 (extremes of normative data) where clinical decisions are usually made, it can be simulated that CPS may not always be fulfilled.

As an illustration we applied the Bidlingmaier normative data to calculate SDS (6) for two fictional clinical cases. The assay and SDS variation were simulated in relation to the MU and CPS at different targets ( 0 S.D., -2 s.D. and +2 s.D.). The MU of the IGF-1 assay of $22 \%$ does not meet CPS criteria in a 8-year-old male for IGF-1 concentrations at +2 and slightly around -2 s.D., which represent clinical decision limits (Fig. 2A). In a male aged 50 years the CPS criteria were not met at IGF-1 concentrations around either $-2,0$ or +2 s.D. (Fig. 2B). As MU of IGF-1 in 2018 had improved to $16 \%$, in these two cases CPS were now fulfilled at most SDS cut off points. The same graphs can be made for every age/gender group.

The development of a reference method for IGF-1 is a complex time-consuming task usually coordinated from the international federation of clinical chemistry (IFCC). Since a reference method will not be available on short term, efforts are made in the Netherlands to improve harmonization among assays. Recently, a LC-MS/MS mass spectrometry method (normalized to $02 / 254 \mathrm{WHO}$ standard) was developed (11). It will be used to determine new reference values (samples will comprise a set from the so-called Lifeline population survey, a large cohort of healthy individuals). Besides, this method is aimed to be used as a designated comparison method, as it is based on analyte-specific physicochemical properties and therefore less influenced by issues associated with immunoassay use (such as sensitivity to interferences, dependency to antibodies used etc.). In the Netherlands, IGF-1 immunoassays (now all traceable to WHO 02/254) are since 2013 harmonized with the harmonization sample pool with concentration of $23 \mathrm{nmol} / \mathrm{L}$. The harmonization appeared not sufficient due to use of a single sample for harmonization. Therefore, the samples with different concentrations of IGF-1 have to be collected. Their target values will be assigned by this designated LC-MS/MS method. The use of different sample pools may lead to more efficient harmonization. This will in turn lead to the lowering of the MU. The lower MU will have less effect on SDS.

In conclusion, when restricted to one single method and a single appropriate set of reference values, the MU of our IGF-1 assay (IDS-iSYS) of 16\% mostly fulfills the clinical performance specification of $+/-0.5$ SDS.
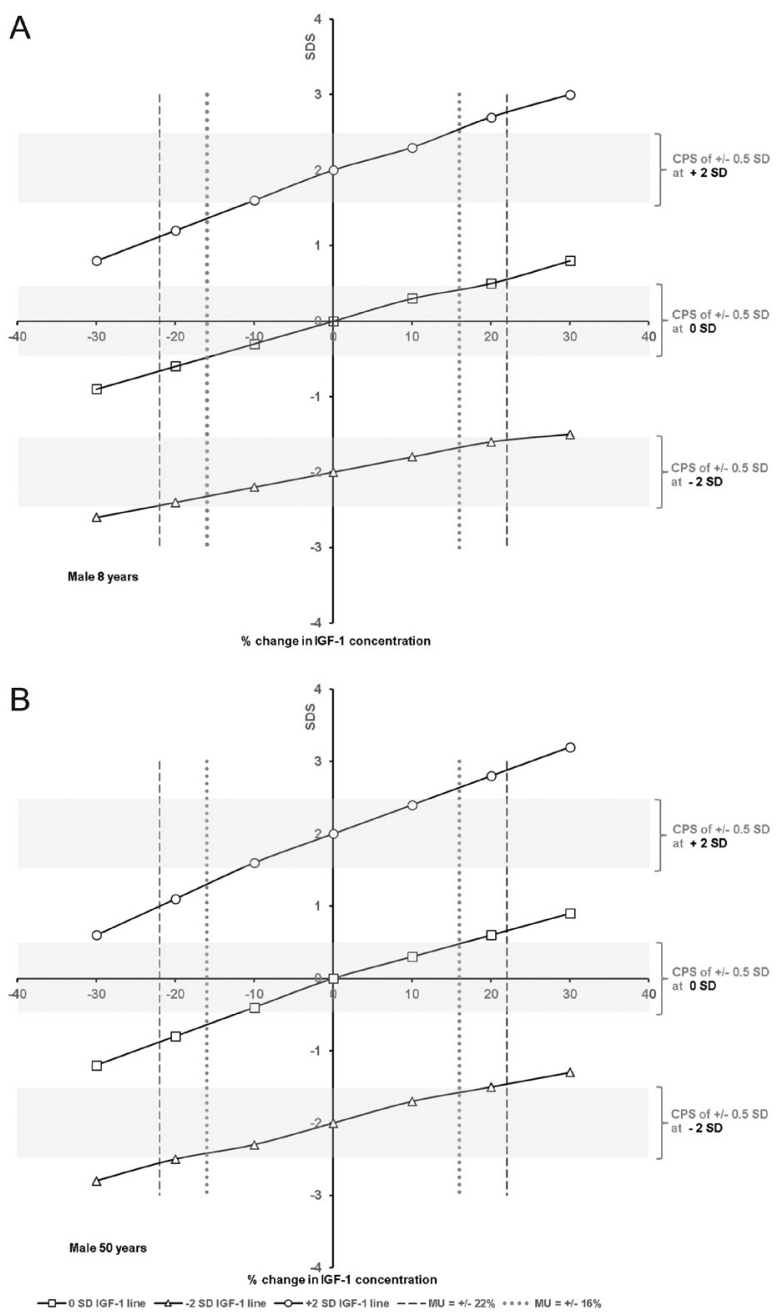

Figure 2

Simulated results presenting the relationship between analytical and clinical performance specifications for IGF-1 and IGF-1 SDS. (A) Simulation in a male, 8 years old; (B) Simulation in a male, 50 years old Maximal methodological variability expressed as measurement uncertainty (MU) was determined to be $\pm 22 \%$ (dashed line) and $\pm 16 \%$ (dotted line) in respectively 2017 and 2018 for IGF-1 concentration range from 8.7 to $59.2 \mathrm{nmol} / \mathrm{L}$. The gray area shows clinical performance specifications (CPS) limits of target expressed as \pm 0.5 s.D. The $\mathrm{x}$-axis represents for a given age/gender group, the possible methodological variability in IGF-1 concentration compared to IGF-1 concentrations at 0 S.D. $(-\square-),+2$ S.D. $(-\infty-)$ and -2 s.D. targets $(-\backsim)$, respectively. The three lines in both figures fall outside the gray area at different \% change from target IGF-1 concentration illustrating non-linear relationship between IGF-1 concentration and SDS. CPS, clinical performance specifications meaning maximal allowable deviation from target SD(S); MU, measurement uncertainty of IGF-1 assay; $\mathrm{SD}(\mathrm{S})$, standard deviation (score). 
Any added variation like inter-method variation or use of various reference value sets will increase the MU beyond this acceptable performance specification. There is a need for further standardization to be able to compare IGF-1 SDS between laboratories. Once available, new calculations must demonstrate that the MU steadily meets the CPS criteria. The endocrinology expert's 'feeling', clinical context and knowledge of the assay used are crucial for the interpretation of the IGF-1 results.

\section{Declaration of interest:}

The authors declare that there is no conflict of interest that could be perceived as prejudicing the impartiality of the research reported.

\section{Funding}

This research did not receive any specific grant from any funding agency in the public, commercial or not-for-profit sector.

\section{References}

1 Varewijck AJ, van der Lely AJ, Neggers SJCMM, Hofland LJ \& Janssen JAMJL. Disagreement in normative IGF-I levels may lead to different clinical interpretations and GH dose adjustments in GH deficiency. Clinical Endocrinology $2018 \mathbf{8 8}$ 409-414. (https://doi. org/10.1111/cen.13491)

2 Mavromati M, Kuhn E, Agostini H, Brailly-Tabard S, Massart C, Piketty ML, Arnoux A, Young J, Souberbielle JC \& Chanson P. Classification of patients with GH disorders may vary according to the IGF-I assay. Journal of Clinical Endocrinology and Metabolism 2017 102 2844-2852. (https://doi.org/10.1210/jc.2017-00202)

3 Broeren MAC, Krabbe JG, Boesten LS, Hokken-Koelega ACS \& de Rijke YB. Impact of the choice of IGF-I assay and normative dataset on the diagnosis and treatment of growth hormone deficiency in children. Hormone Research in Paediatrics 201890 181-189. (https://doi.org/10.1159/000493133)
4 Clemmons DR. Consensus statement on the standardization and evaluation of growth hormone and insulin-like growth factor assays. Clinical Chemistry 201157 555-559. (https://doi.org/10.1373/ clinchem.2010.150631)

5 Chanson P, Arnoux A, Mavromati M, Brailly-Tabard S, Massart C, Young J, Piketty ML, Souberbielle JC \& VARIETE Investigators. Reference values for IGF-I serum concentrations: comparison of six immunoassays. Journal of Clinical Endocrinology and Metabolism 2016 101 3450-3458. (https://doi.org/10.1210/jc.2016-1257)

6 Bidlingmaier M, Friedrich N, Emeny RT, Spranger J, Wolthers OD, Roswall J, Korner A, Obermayer-Pietsch B, Hubener C, Dahlgren J et al. Reference intervals for insulin-like growth factor-1 (IGF-i) from birth to senescence: results from a multicenter study using a new automated chemiluminescence IGF-I immunoassay conforming to recent international recommendations. Journal of Clinical Endocrinology and Metabolism 201499 1712-1721. (https://doi.org/10.1210/jc.2013-3059)

7 Ross HA \& Endocrinology section and project group "Calibration 2000" of the SKML (Dutch Foundation for Quality Assessment in Clinical Laboratories). Reporting growth hormone assay results in terms of one consensus recombinant standard preparation offers less than optimal reduction of between-method variation. Clinical Chemistry and Laboratory Medicine 200846 1334-1335. (https://doi. org/10.1515/CCLM.2008.261)

8 Ross HA, Lentjes E \& Menheere PP. The consensus statement on the standardization and evaluation of growth hormone and insulin-like growth factor assays lacks a recommendation to attempt efficacious harmonization. Clinical Chemistry 201157 1463; author reply 1463-1464. (https://doi.org/10.1373/clinchem.2011.169771)

9 Horvath AR, Bossuyt PM, Sandberg S, John AS, Monaghan PJ, Verhagen-Kamerbeek WD, Lennartz L, Cobbaert CM, Ebert C, Lord SJ et al. Setting analytical performance specifications based on outcome studies - is it possible? Clinical Chemistry and Laboratory Medicine 201553 841-848. (https://doi.org/10.1515/cclm-2015-0214)

10 Algeciras-Schimnich A, Bruns DE, Boyd JC, Bryant SC, La Fortune KA $\&$ Grebe SK. Failure of current laboratory protocols to detect lot-to-lot reagent differences: findings and possible solutions. Clinical Chemistry 201359 1187-1194. (https://doi.org/10.1373/ clinchem.2013.205070)

11 Bronsema KJ, Klont F, Schalk FB, Bischoff R, Kema IP \& van de Merbel NC. A quantitative LC-MS/MS method for insulin-like growth factor 1 in human plasma. Clinical Chemistry and Laboratory Medicine 201856 1905-1912. (https://doi.org/10.1515/cclm-2017-1042)

Received 25 July 2019

Revised version received 30 August 2019

Accepted 9 September 2019 